\title{
Restructuring of the electrical double layer in ionic liquids upon charging
}

\author{
V. Ivaništšev \\ Department of Physics, Scottish Universities Physics Alliance (SUPA), Strathclyde University, \\ John Anderson Building, 107 Rottenrow East, Glasgow, UK G4 0NG. \\ E-mail: vladislav.ivanistsev@strath.ac.uk
}

\section{K. Kirchner}

\section{T. Kirchner}

Department of Physics, Scottish Universities Physics Alliance (SUPA), Strathclyde University, John Anderson Building, 107 Rottenrow East, Glasgow, UK G4 0NG.

Max Planck Institute for Mathematics in the Sciences, Inselstraße 22, 04103 Leipzig, Germany.

\author{
M.V. Fedorov \\ Department of Physics, Scottish Universities Physics Alliance (SUPA), Strathclyde University, \\ John Anderson Building, 107 Rottenrow East, Glasgow, UK G4 0NG. \\ E-mail: maxim.fedorov@strath.ac.uk
}

\begin{abstract}
We have investigated the electrical double layer (EDL) structure in ionic liquids using molecular dynamics simulations with three different models of ionic liquids. We show that the EDL restructuring, driven by surface charging, can be rationalized by use of two parameters, renormalized surface charge $(\kappa)$ and charge excess in interfacial layers $(\lambda)$. Analysis of relationships between the $\lambda$ and $\kappa$ parameters provides new insights into mechanisms of over-screening and charge-driven structural transitions in the EDL in ionic liquids. We show that the restructuring of the EDL upon charging in all three studied systems has two characteristic regimes: $(1)$ Transition from the bulk-like $\left(\kappa_{\text {Ion }}=0\right)$ to the multilayer structure $\left(\kappa_{\text {Ion }} \approx 0.5\right)$ through the formation of the commensurate multilayer of counter- and co-ions; and $(2)$ Transition from the multilayer $\left(\kappa_{\text {Ion }} \approx 0.5\right)$ to the crowded $\left(\kappa_{\text {Ion }}>1\right)$ structure through the formation of a monolayer of counter-ions at $\kappa_{\text {Ion }} \approx 1.0$.
\end{abstract}

Research on ionic liquids (ILs) ${ }^{1}$ at charged interfaces is booming due a large number of existing and prospective applications of these systems (particularly of room temperature ILs) in energy, metal extraction, catalysis and many other fields $[1,2,3]$. ILs are interesting systems from the fundamental point of view as well because they represent an unusual case of highly concentrated electrolytes that in addition are generally more chemically inert and more resistant to higher voltages than standard diluted electrolytes (such as e.g. aqueous ionic solutions) $[1,4]$. By this time, it has been already realised that the behaviour of ILs at charged surfaces is

1 By an ionic liquid we mean a fluid system that is composed solely from anions and cations without any solvent. 
principally different to the behaviour of standard solvent-based electrolytes: ILs reveal a number of phenomena typically not seen in standard electrolytes such as over-screening [5, 6], chargedriven structural transitions [7,8] and ion crowding at the interface [9] (for a review of these phenomena, see Ref.[1]).

Most of these phenomena are determined by the structural response of the IL electrical double layer (EDL) ${ }^{2}$ to the surface charge [1]. The aim of this paper is to elucidate main mechanisms of the charge-driven changes of the EDL structure in ILs by investigating interfacial behaviour of three different model ILs in a wide range of surface charge densities. To facilitate comparison of different IL systems and reveal general trends in structural behaviour of ILs at charged interfaces we perform analysis of the EDL structure and capacitance on the base of two dimensionless parameters, renormalized surface charge $(\kappa)$ and charge excess in interfacial layers $(\lambda)[7,10]$.

Focussing on rationalising the crossover between the overscreening and crowding regimes in the EDL structure [9], we run several extensive series of long-term and large scale simulations for a range of surface charge densities (as wide as $\pm 76 \mu \mathrm{C} \mathrm{cm}^{-2}$ ). In order to elucidate the effect of the ion size, three different charged Lennard-Jones sphere models of IL were chosen, with the cation-to-anion diameter $\left(d_{\mathrm{LJ}}\right)$ ratios of $1: 1$ (large anion - LA), $1: 0.8$ (medium anion - MA) and $1: 0.5$ (small anion $-\mathrm{SA})$ with constant $d_{\mathrm{LJ}}($ Cation $)=1.0 \mathrm{~nm}$. Two of these models (LA and SA) were verified in previous works on ILs at electrified interfaces $[11,12,7]$ where it was shown that they can provide qualitative comparison with experimental data on the EDL capacitance in ILs. All simulations were performed using the GROMACS Molecular Dynamics (MD) software [13]. Preparation of the model systems and analysis of the MD simulations data were held using NaRIBaS toolbox ${ }^{3}$. Further details on the systems and models are provided in the Supplementary Material [14].

In the analysis presented below we investigate behaviour of number and charge densities of IL ions in the interfacial area in order to elucidate relationships between several interfacial phenomena attributed to ILs, including:

- ion layering and charge-density oscillations in the direction that is normal to the charged surface $[12,6]$;

- crowding of counter-ions at high surface charge densities [9];

- overscreening of the surface charge by the charge of counter-ions in the first interfacial layer $[5,12]$.

The overscreening regime is a distinctive feature of the EDL structure in ILs [1]. It is manifested in the formation of a layered structure near the charged electrode surface, where the first layer contains more counter-charge than is needed to compensate for the surface charge [5]. The excess of charge is provided by counter-ions (ions with opposite charge to the surface) and it is compensated in the subsequent ion layers. The term crowding refers to the situation where the number of counter-ions required to compensate the surface charge can not be accommodated in a layer with thickness of a single ion diameter [9]. Therefore, the first ionic layer becomes wider due to inclusion of several sublayers of counter-ions.

As demonstrated below, the crossover between the overscreening and crowding regimes happens via formation of a monolayer of counter-ions (Fig. 1A). The monolayer structure

2 EDL is a common name for a complex interfacial structure in systems with mobile charges determined by the electrostatic interaction of ions and molecules with a charged surface. Despite of having "double" in its name, the EDL may consist of several distinct molecular or charge layers, particularly in highly concentrated electrolytes like ionic liquids (ILs) [1].

${ }^{3}$ K. Kirchner and M.V. Fedorov, NaRIBaS: A scripting framework for computational modeling of Nanomaterials and Room temperature Ionic liquids in Bulk and Slab; Version 1.0 (2013). http://sourceforge.net/projects/naribas/ 
is characterized by an absence of oscillations in the electrostatic potential $\phi(z)$-profiles ${ }^{4}$ and a linear potential drop between the electrode and the monolayer of counter-ions [14]. We employ this feature to define a unified $\kappa$-scale, on which the surface charge density $(\sigma)$ is normalized by the maximum charge density that can be stored in the counter-ion monolayer $\left(\theta_{\mathrm{Ion}}^{\max }\right)$ :

$$
\kappa_{\text {Ion }}=\left|\frac{\sigma}{\theta_{\text {Ion }}^{\max }}\right| .
$$

Below we use the $\kappa$-scale for generalized analysis with a focus on the region $0<\kappa_{\text {Ion }}<1.5$, where $\kappa_{\text {Cation }}$ corresponds to the negative surface charge density values $(\sigma<0)$ and $\kappa_{\text {Anion }}$ corresponds to the positive values $(\sigma>0)$.

The physical basis for the $\kappa$-scale lies in the complete surface charge compensation by the monolayer of counter-ions at $\kappa_{\text {Ion }}=1$, i.e. when $\sigma=-\theta_{\text {Ion }}^{\max }[7,10]$. The latter quantity $\left(\theta_{\text {Ion }}^{\max }\right)$ can be either estimated on the basis of a purely geometrical model of the monolayer (see Fig. 1B) or extracted from the simulation results at the point of surface charge that corresponds to a linear potential drop [14]. Value of $\theta_{\text {Cation }}^{\max }$ was found to be the same for all studied systems $\left(16 \mu \mathrm{C} / \mathrm{cm}^{2}\right)$ in accordance with the fact that the cation model is the same in all systems. This value corresponds to the density of one cation per $1 \mathrm{~nm}^{2}$ of the surface that corresponds to the dense coverage of the surface by the cations (the cation diameter is $1 \mathrm{~nm}$ ). $\theta_{\text {Anion }}^{\max }$ values were found to be $-68,-26$ and $-16 \mu \mathrm{C} / \mathrm{cm}^{2}$ for the systems with small, medium and large anions, respectively. These values also correspond to the dense coverage of the surface by the anions.

In Fig. 1, ion density $\rho_{N}(z)$-profiles are summarized for the model with medium anion in the form of a contour map of $\rho_{N}(z, \kappa)^{5}$. As can be seen, the vertical valleys divide the interfacial region into distinct regions of ion accumulation. Accordingly, we refer to the region of accumulation of counter-ions as the first layer and the subsequent region of co-ions as the second interfacial layer.

Upon surface charging, i.e. increasing $\kappa_{\text {Ion }}$, counter-ions in the first layer become pressed against the surface by the strong electrostatic attraction between the ions and the charged surface. This is evident from the thinning of the first layer and the monotonous grow of the counter-ion number density with increasing $\kappa_{\text {Ion }}$ (Fig. 1). Differently, in the second layer the $\rho_{N}(z, \kappa)$ grows until some saturation at $\kappa_{\text {Ion }} \approx 0.5$ and then decreases until formation of the monolayer at $\kappa_{\text {Ion }}=1$. The highlighted horizontal valleys indicate an absence of layering and a direct transition from the monolayer of counter-ions to bulk-like IL in direction normal to the surface plane (Fig. 1A).

Note that the maximum number density, seen at $\kappa_{\text {Ion }} \approx 0.5$, considerably exceeds the bulk number density $\left(\rho_{\text {bulk }}\right)$. This implies more organized packing and also indicates that the most distinct layering occurs in the vicinity of $\kappa_{\text {Ion }} \approx 0.5$. The conclusion is supported by the appearance of a flat peak in the normalized cumulative charge density $\operatorname{cn}_{Q}(z)$-profiles: $\operatorname{cn}_{Q}(z)=\int_{0}^{z} \rho_{Q}\left(z^{\prime}\right) \mathrm{d} z^{\prime}$, where the charge density $\rho_{Q}(z)$-profile is defined as a sum of the ion number density profiles multiplied by the charge of the ionic species.

The $-\mathrm{cn}_{Q}(z) / \sigma$-profiles, shown in Fig. 2, resemble waves with crests that is a clear indicator the overscreening regime $\left(\left|\operatorname{cn}_{Q}(z) / \sigma\right|>1\right)$. Upon $\kappa_{\text {Ion }} \rightarrow 1$ the overscreening diminishes gradually. At $\kappa_{\text {Ion }}=1$ the profiles represent a step-wise curve suddenly reaching value of 1.0, and in the crowding regime at $\kappa>1.0$ the overscreening reappears. The absence of the charge waves at $\kappa_{\text {Ion }}=1$ confirms that in the surface normal direction the monolayer is directly followed by the bulk-like phase. Note that the distinct layering and oscillations are visible in both

4 The EDL structural response to the surface charging is commonly analysed through calculations of electrostatic potential profiles $-\phi(z)$, ion number density profiles $-\rho_{N}(z)$ or charge density profiles $-\rho_{Q}(z)$, which depict the dependence of the corresponding quantity on the distance from the surface $(z)$

5 Ion densities for other models (not shown) behave in a similar way. 
Fig. 1 and Fig. 2. However, variations of $\rho_{\mathrm{N}}(z)$ - and $-\operatorname{cn}_{Q}(z) / \sigma$-profiles hinder several essential features of the EDL restructuring. More clearly the restructuring process can be illustrated with the use of a charge excess $(\lambda)$ in an $i$-th ion layer [10]:

$$
\lambda_{i}=\kappa_{\mathrm{Ion}} \times\left(\left|\frac{\mathrm{cn}_{Q}\left(z_{i}\right)}{\sigma}\right|-1\right)
$$

where $z_{i}$ corresponds to an extremum or to a step height on the $-\mathrm{cn}_{Q}(z) / \sigma$-profiles in an $i$-th interval between two successive interception points $\left|\mathrm{cn}_{Q}(z) / \sigma\right|=0$. In Fig. 3 the positive charge excess values $\left(\lambda_{1}\right)$ correspond to the first maxima in the $-\operatorname{cn}_{Q}(z) / \sigma$-profiles, which is determined by the accumulation of counter-ions in the first interfacial layer. The negative values of $\left(\lambda_{2}\right)$ correspond to the following minima and are related to the accumulation of co-ions in the second layer.

We note that the screening factor $\left|\mathrm{cn}_{Q}(z) / \sigma\right|$ gradually becomes a common parameter for characterising the EDL structure in ILs $[12,15,16]$. Besides, the conception of charge magnitude, similar to ours, was previously applied in analysis of atomistic simulation results in Ref. [15]. However, different from the previous studies, in this work we present results of systematic variation of $\lambda_{i}$ over a much larger set of (renormalized) surface charge densities (see Fig. 3).

The $\lambda_{i}-\kappa_{\text {Ion }}$ dependence represents a universal analogue of dimensionless "reaction coordinate" for the EDL restructuring process. It allows the tracking of the evolution of the EDL structure in terms of the charge excess. Namely, with increase of $\kappa_{\text {Ion }}$ the increase of the charge excess in the first interfacial layer $\left(\lambda_{1}\right)$ manifests transition from the bulk-like to the multilayer EDL structure, while the decrease of the charge excess indicates the vanishing of the multilayer EDL structure towards exposure of the monolayer structure at $\kappa_{\text {Ion }}=1$.

The bend on the $\lambda_{i}$-curves (see Fig. 3) implies slowdown of the accumulation of counterions in the first layer and decrease in the number of the co-ions in the second layer upon increasing $\kappa_{\text {Ion }}$. The slowdown is apparently related to a crossover of the two mechanisms of ion accumulation at the surface: specific packing of ions determined by the strong anion-cation correlation $\left(\kappa_{\text {Ion }} \rightarrow 0.5\right)$ and electrostriction of the first layer $\left(\kappa_{\text {Ion }} \rightarrow 1\right){ }^{6}$. The first mechanism is similar to the so called "lattice saturation" mechanism of ion packing in ILs at charged surfaces $[5,9,17, ?]$. However, differing from the original "saturation" concept it is related to the maximum number density of co-ions, not counter-ions (see Fig. 1). We suggest that the above-mentioned specific packing at $\kappa_{\text {Ion }} \approx 0.5$ is a commensurate multilayer, in which some ions are arranged in a pseudo lattice (in the "spirit" of Ref. [18]). By means of breaking the anion-cation correlation the electrostriction mechanism [17] allows for more dense packing of counter-ions in a form of the monolayer $\left(\kappa_{\text {Ion }} \rightarrow 1\right)$.

Both mechanisms are related to the overscreening phenomenon. We speculate that the overscreening originates from a tendency of counter-ions to preserve their ion cage structures while entering the first interfacial layer [19]. When counter-ions approach a charged surface, their cage structures transform into the second interfacial layer and cause the formation of the subsequent interfacial layers [20]. In accordance with the electroneutrality principle, the excess of counter-ions in the first layer is induced by the strong anion-cation interaction, on one side, and the electrode-counter-ion interaction, on the other. Thus, the interplay between short-range electrostatic interactions and steric effects results in the ion layering (Fig. 1), the overscreening (Fig. 2), and the accumulation of the excess charge in the ion layers (Fig. 3) [9].

In the region $0<\kappa_{\text {Ion }}<0.5$ this interplay also leads to the appearance of peaks at the differential capacitance $(C)$ dependence on $\kappa_{\text {Ion }}$ (Fig. 4). A detailed explanation of this structure-property relation is beyond of the scope of this work and will be considered in detail

${ }^{6}$ By electrostriction we mean local increase of the ion density at the surface due to their strong electrostatic attraction to the surface [17]. 
elsewhere. In this letter we turn the readers attention to the dependence of $C$ on $\kappa_{\text {Ion }}$ close to the potential of the monolayer formation.

With the focus on the crossover between the overscreening and crowding regimes, we would like to discuss how this interplay determines the dependence of differential capacitance $(C)$ on $\kappa_{\text {Ion }}$, close to the potential of the monolayer formation. The capacitance curves presented on Fig. 4 show that above $\kappa_{\text {Cation }} \approx 0.5$, the dependence of $C$ on $\kappa_{\text {Cation }}$ coincides for all studied systems, presumably due to the common cation. This observation implies that above $\kappa_{\text {Ion }} \approx 0.5$ counter-ions of the first interfacial layer completely govern the response of the EDL to the charging of a surface. The next feature is the (approximate) inverse proportionality of $C$ values on the anion diameter, close to $\kappa_{\text {Anion }}=1$.

The basis of this structure-property relationship is the above described complete surface charge compensation by the monolayer of counter-ions. The monolayer structure observed for the coarse-grained models is the simplest form of a compact layer that can be seen in simulations with different IL models at the crossover from overscreening to crowding regimes [14]. Presumably, more complex compact structures can be formed in ILs with complex molecular geometries of ions (e.g. ions with long non-polar tails). The effects of molecular geometry of the ions on the structural reorganisation in the EDL will be considered in our future works.

An experimental confirmation of the compact structure formation is likely to be found for novel ILs with very large ions, specifically adsorbing halogenides or large multivalent counterions, like $\left[\mathrm{PW}_{12} \mathrm{O}_{40}\right]^{3-}[21, ?, ?]$. It is tempting to declare that the monolayer structure has already been observed via scanning tunneling microscopy in 1-butyl-3-methylimidazolium hexafluorophosphate at $\mathrm{Au}(111)$ electrode [22]. However, a careful comparison of similar experimental studies [23, ?] assures us to assign the demonstrated hexagonal pattern of the probable $\left[\mathrm{PF}_{6}\right]^{-}$monolayer to a less dense structure forming in our simulations at $\kappa_{\text {Ion }} \approx 0.5$.

To summarise, in this letter we show that the restructuring of the EDL in ILs has two characteristic crossover regions on the $\kappa$-scale: (1) Transition from the bulk-like $\left(\kappa_{\text {Ion }}=0\right)$ to the multilayer structure $\left(\kappa_{\text {Ion }} \approx 0.5\right)$ through the formation of the commensurate multilayer of counter- and co-ions; and $(2)$ Transition from the multilayer $\left(\kappa_{\text {Ion }} \approx 0.5\right)$ to the crowded $\left(\kappa_{\text {Ion }}>1\right)$ structure through the formation of the monolayer of counter-ions at $\kappa_{\text {Ion }} \approx 1.0$. The presented results reveal that the evolution of the ion layering $\left(\rho_{\mathrm{N}}(\right.$ Ion $)$ in Fig. 1$)$, the overscreening $\left(-\operatorname{cn}_{Q}(z) / \sigma\right.$ in Fig. 2) and the charge excess in interfacial layers $(\lambda$ in Fig. $3)$ represents a "wave-like" pattern on the $\kappa$-scale. The results of our work can be used for predicting and control of ion layering in IL-based interfacial systems. This can be important for various electrochemical and electromechanical applications of ILs [1] because it has been shown recently that the ion layering determines the free energy barriers for a solute approaching the electrode $[24, ?]$ and the potential-dependent variation of nanoscale-level friction at the IL-electrode interfaces $[25, ?]$.

We are grateful for the large amount of computer time provided by the EPSRC-funded ARCHIE-WeSt High Performance Computer Centre (www.archie-west.ac.uk, EPSRC grant no. EP/K000586/1) and for the supercomputing support from the von Neumann-Institut für Computing, FZ Jülich (Project ID ESMI11).

\section{References}

[1] Fedorov M V and Kornyshev A A 2014 Chem. Rev. 114 2978-3036

[2] Seddon K R and Plechkova N V 2012 Ionic Liquids UnCOILed: Critical Expert Overviews (John Wiley \& Sons)

[3] Endres F, Höfft O, Borisenko N, Gasparotto L H, Prowald A, Al-Salman R, Carstens T, Atkin R, Bund A and El Abedin S Z 2010 Phys. Chem. Chem. Phys. 12 1724-1732

[4] Galiński M, Lewandowski A and Stepniak I 2006 Electrochim. Acta 51 5567-5580

[5] Kornyshev A A 2007 J. Phys. Chem. B 111 5545-5557 
[6] Mezger M, Schröder H, Reichert H, Schramm S, Okasinski J S, Schöder S, Honkimäki V, Deutsch M, Ocko B M, Ralston J, Rohwerder M, Stratmann M and Dosch H 2008 Science 322 424-428

[7] Kirchner K, Kirchner T, Ivaništšev V and Fedorov M 2013 Electrochim. Acta $110762-771$

[8] Atkin R, Borisenko N, Drüschler M, Endres F, Hayes R, Huber B and Roling B 2014 J. Mol. Liq. 192 44-54

[9] Bazant M Z, Storey B D and Kornyshev A A 2011 Phys. Rev. Lett. 106 046102-4

[10] Ivaništšev V and Fedorov M V 2014 Interface 23 65-69

[11] Fedorov M V and Kornyshev A A 2008 J. Phys. Chem. B 112 11868-11872

[12] Fedorov M V and Kornyshev A A 2008 Electrochim. Acta 53 6835-6740

[13] Pronk S, Páll S, Schulz R, Larsson P, Bjelkmar P, Apostolov R, Shirts M R, Smith J C, Kasson P M, Spoel D v d, Hess B and Lindahl E 2013 Bioinformatics 29 845-854

[14] See Supplementary Material

[15] Feng G, Huang J, Sumpter B G, Meunier V and Qiao R 2011 Phys. Chem. Chem. Phys. 13 14723-14734

[16] Paek E, Pak A J and Hwang G S 2013 J. Electrochem. Soc. 160 A1-A10

[17] Georgi N, Kornyshev A A and Fedorov M V 2010 J. Electroanal. Chem. 649 261-267

[18] Varela L M, Carrete J, García M, Rodríguez J R, Gallego L J, Turmine M and Cabeza O 2011 Ionic Liquids: Theory, properties, new approaches ed Kokorin A (Shanghai: InTech) pp 347-366

[19] Ivaništšev V, Fedorov M V and Lynden-Bell R M 2014 J. Phys. Chem. C $1185841-5847$

[20] Yochelis A 2014 Phys. Chem. Chem. Phys. 16 2836-2841

[21] Cuvillier N and Rondelez F 1998 Thin Solid Films 327-329 19-23

[22] Pan G B and Freyland W 2006 Chem. Phys. Lett. 427 96-100

[23] Zhang X, Zhong Y X, Yan J W, Su Y Z, Zhang M and Mao B W 2012 Chem. Commun. 48 582-584

[24] Lynden-Bell R M, Frolov A I and Fedorov M V 2012 Phys. Chem. Chem. Phys. 14 2693-2701

[25] Sweeney J, Hausen F, Hayes R, Webber G B, Endres F, Rutland M W, Bennewitz R and Atkin R 2012 Phys. Rev. Lett. 109 155502-5 


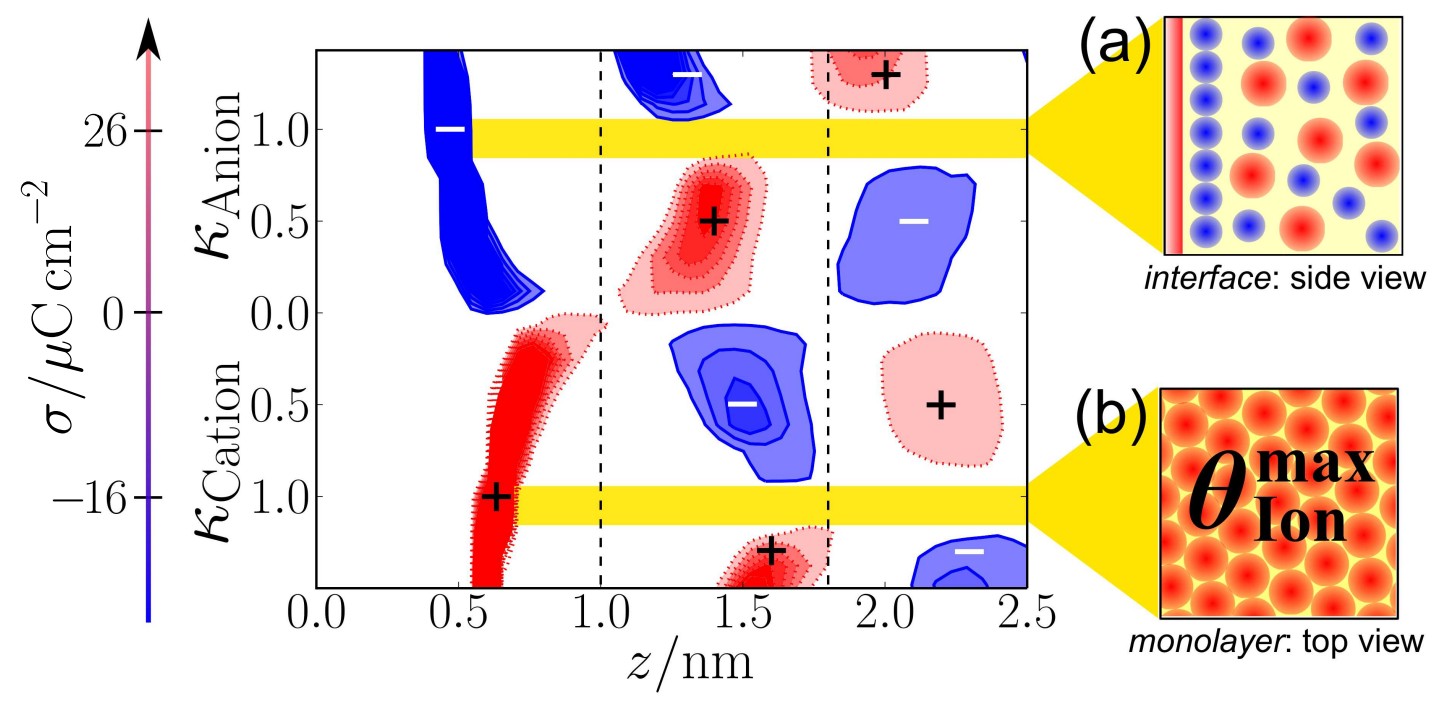

Figure 1. The contour map illustrates the dependence of the cation (red) and anion (blue) density at the interface on $\kappa, \rho_{N}(z, \kappa)$. The data are shown for the model system with medium anion (MA). The contour interval equals to the bulk density of the IL system, $\rho_{\text {bulk }}$; the first contour starts at $1.5 \rho_{\text {bulk }}$, and the maximum peaks of $\rho_{N}(z, \kappa)$ are cut at $7 \rho_{\text {bulk }}$ to facilitate the visual analysis. The map clearly indicates changes of the ion layering with $\kappa$, the formation of cation/anion monolayers at $\kappa$-values close to 1.0 and interfacial crowding of ions at higher $\kappa$-values. Insets: (a) schematic representation of a monolayer of anions at the positively charged surface at $\kappa_{\text {Anion }}=1$ and the bulk-like structured IL close to the monolayer; (b) schematic representation of a monolayer of cations at $\kappa_{\text {Cation }}=1$ with the sterically determined maximum charge density $\left(\theta_{\mathrm{Ion}}^{\max }\right)$. 


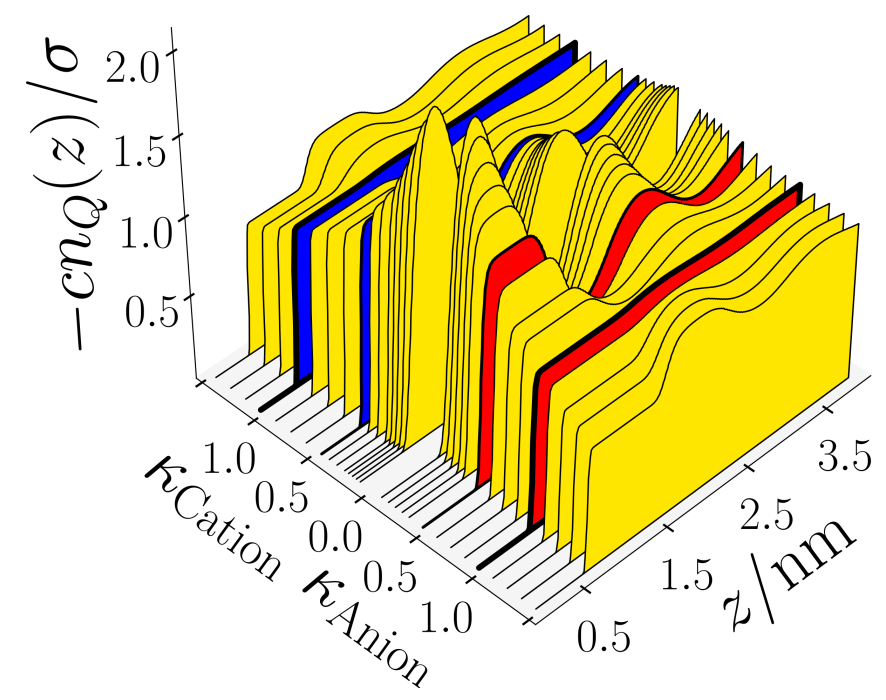

Figure 2. $-c n_{Q}(z) / \sigma$-profiles illustrate the surface charge (over)screening on the $\kappa$-scale for the model system with medium anion. Profiles at $\kappa_{\text {Ion }}$ values of 0.5 and 1.0 are highlighted to underline the vanishing of the charge-density oscillations at $\kappa$-values close to 1.0.

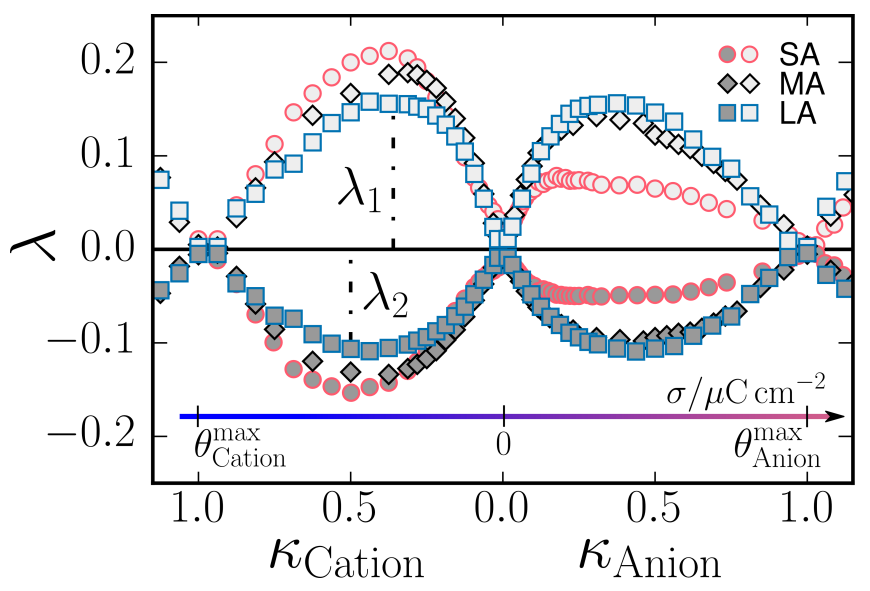

Figure 3. Variation of the charge excess in the first $\left(\lambda_{1}\right.$, lighter marks) and in the second $\left(\lambda_{2}\right.$, darker marks) interfacial layers illustrates the EDL restructuring upon surface charging: from a bulk-like $\left(\kappa_{\text {Ion }} \rightarrow 0, \lambda_{i} \rightarrow 0\right)$ to the multilayer EDL structure $\left(\kappa_{\text {Ion }} \approx 0.5,\left|\lambda_{i}\right|>0\right)$; from the multilayer to the monolayer EDL structure $\left(\kappa_{\text {Ion }} \rightarrow 1, \lambda_{i} \rightarrow 0\right)$; and from the monolayer to the crowded EDL structure $\left(\kappa_{\text {Ion }}>1,\left|\lambda_{i}\right|>0\right)$. The data are shown on the $\kappa$-scale for the model systems with small $(\circ, \mathrm{SA})$, medium $(\diamond, \mathrm{MA})$ and large $(\square, \mathrm{LA})$ anions. The $\kappa_{\text {Ion }}$ values of 1.0 correspond to the $\theta_{\mathrm{Ion}}^{\max }$ values of the charge density $\sigma$ (see the $\sigma$-scale that is shown in the bottom). 


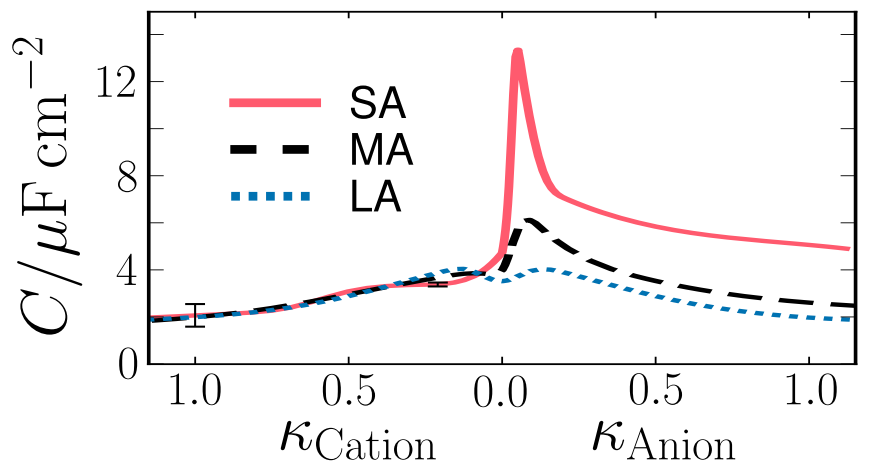

Figure 4. Dependence of the EDL differential capacitance $C$ on $\kappa_{\text {Ion }}$ for the model systems with small (solid line, SA), medium (dashed line, MA) and large (dotted line, LA) anion. Error bars represent the standard deviation of the capacitance calculations which steadily increases with increasing $\kappa_{\text {Ion }}$ from $\pm 0.1 \mathrm{~F} \mathrm{~cm}^{-2}$ to $\pm 0.5 \mathrm{~F} \mathrm{~cm}^{-2}$ (indicated in the plot). 